\title{
SUBLINEAR EQUATIONS AND SCHUR'S TEST FOR INTEGRAL OPERATORS
}

\author{
IGOR E. VERBITSKY \\ In memory of Professor Victor Havin
}

ABSTRACT. We study weighted norm inequalities of $(p, r)$-type,

$$
\|\mathbf{G}(f d \sigma)\|_{L^{r}(\Omega, d \sigma)} \leq C\|f\|_{L^{p}(\Omega, \sigma)}, \quad \text { for all } f \in L^{p}(\sigma),
$$

for $0<r<p$ and $p>1$, where $\mathbf{G}(f d \sigma)(x)=\int_{\Omega} G(x, y) f(y) d \sigma(y)$ is an integral operator associated with a nonnegative kernel $G(x, y)$ on $\Omega \times \Omega$, and $\sigma$ is a locally finite positive measure in $\Omega$.

We show that this embedding holds if and only if

$$
\int_{\Omega}(\mathbf{G} \sigma)^{\frac{p r}{p-r}} d \sigma<+\infty
$$

provided $G$ is a quasi-symmetric kernel which satisfies the weak maximum principle.

In the case $p=\frac{r}{q}$, where $0<q<1$, we prove that this condition characterizes the existence of a non-trivial solution (or supersolution) $u \in L^{r}(\Omega, \sigma)$, for $r>q$, to the the sublinear integral equation

$$
u-\mathbf{G}\left(u^{q} d \sigma\right)=0, \quad u \geq 0 .
$$

We also give some counterexamples in the end-point case $p=1$, which corresponds to solutions $u \in L^{q}(\Omega, \sigma)$ of this integral equation, studied recently in [19], [20]. These problems appear in the investigation of weak solutions to the sublinear equation involving the (fractional) Laplacian,

$$
(-\Delta)^{\alpha} u-\sigma u^{q}=0, \quad u \geq 0
$$

for $0<q<1$ and $0<\alpha<\frac{n}{2}$ in domains $\Omega \subseteq \mathbb{R}^{n}$ with a positive Green function.

\section{INTRODUCTION}

Let $\Omega$ be a locally compact, Hausdorff space. For a positive, lower semicontinuous kernel $G: \Omega \times \Omega \rightarrow(0,+\infty]$, we denote by

$$
\mathbf{G}(f d \sigma)(x)=\int_{\Omega} G(x, y) f(y) d \sigma(y), \quad x \in \Omega,
$$

the corresponding integral operator, where $\sigma \in \mathscr{M}^{+}(\Omega)$, the class of positive locally finite Radon measures in $\Omega$.

We study the $(p, r)$-weighted norm inequalities

$$
\|\mathbf{G}(f d \sigma)\|_{L^{r}(\Omega, \sigma)} \leq C\|f\|_{L^{p}(\Omega, \sigma)}, \quad \forall f \in L^{p}(\Omega, \sigma),
$$

2010 Mathematics Subject Classification. Primary 35J61, 42B37; Secondary 31B15, 42B25.

Key words and phrases. Weighted norm inequalities, sublinear elliptic equations, weak maximum principle, Green's function, fractional Laplacian. 
in the case $0<r<p$ and $p \geq 1$, where $C$ is a positive constant which does not depend on $f$.

The main goal of this paper is to find explicit characterizations of (1.1) in terms of $\mathbf{G} \sigma$ under certain assumptions on $G$. We also study connection of inequality (1.1) with $p=\frac{r}{q}$, where $0<q<1$, to the existence of a positive function $u \in$ $L^{r}(\Omega, \sigma)$ such that

$$
u \geq \mathbf{G}\left(u^{q} \sigma\right) \quad d \sigma-\text { a.e. in } \Omega,
$$

in the case $r>q$. In other words, $u$ is a supersolution for the sublinear integral equation

$$
u-\mathbf{G}\left(u^{q} \sigma\right)=0, \quad 0<u<+\infty \quad d \sigma-\text { a.e. in } \Omega,
$$

where $0<q<1$.

In this paper, we assume that the kernel $G$ of the integral operator is quasisymmetric, and satisfies a weak maximum principle (WMP); see Sec. 2 Such restrictions are satisfied by the Green kernel associated with many elliptic operators, including the fractional Laplacian $(-\Delta)^{\alpha}$, as well as quasi-metric kernels, and radially symmetric, decreasing convolution kernels $G(x, y)=k(|x-y|)$ on $\mathbb{R}^{n}$ (see, e.g., [1], [2], [18], [19], [20] and the literature cited there).

If $G$ is Green's kernel associated with the Laplacian in an open domain $\Omega \subseteq \mathbb{R}^{n}$, (1.3) is equivalent to the sublinear elliptic boundary value problem

$$
\left\{\begin{array}{lc}
-\Delta u-\sigma u^{q}=0, & u>0 \text { in } \Omega \\
u=0 & \text { on } \partial \Omega
\end{array}\right.
$$

where $0<q<1$.

We observe that solutions $u \in L^{r}(\Omega, \sigma)$ to (1.4) in the case $r=1+q$ correspond to finite energy solutions $u \in L_{0}^{1,2}(\Omega)$ in the Dirichlet space, i.e.,

$$
\int_{\Omega}|\nabla u|^{2} d x<+\infty
$$

where $u$ has zero boundary values (see [5]).

The more difficult end-point case $p=1$ of (1.1), along with solutions $u \in$ $L^{q}(\Omega, \sigma)$ in the case $r=q$, was studied recently in [19], [20]. After a certain modification, it leads to solutions $u \in L_{\mathrm{loc}}^{q}(\Omega, \sigma)$, i.e., all solutions to (1.3), or (1.4) understood in a weak sense (see [16]). For Riesz kernels on $\Omega=\mathbb{R}^{n}$ such $(1, q$ )weighted norm inequalities, along with weak solutions to the sublinear problem

$$
\left\{\begin{array}{l}
(-\Delta)^{\alpha} u-\sigma u^{q}=0, \quad u>0 \text { in } \mathbb{R}^{n}, \\
\liminf _{x \rightarrow \infty} u=0, \quad u \in L_{\mathrm{loc}}^{q}(\sigma),
\end{array}\right.
$$

for $0<\alpha<\frac{n}{2}$, were treated earlier in [5], [6], [7].

Our main result is the following theorem.

Theorem 1.1. Let $\sigma \in \mathscr{M}^{+}(\Omega)$. Suppose $G$ is a positive, quasi-symmetric, lower semicontinuous kernel on $\Omega \times \Omega$ which satisfies the weak maximum principle. 
(i) If $1<p<+\infty$ and $0<r<p$, then the $(p, r)$-weighted norm inequality (1.1) holds if and only if

$$
\int_{\Omega}(\mathbf{G} \sigma)^{\frac{p r}{p-r}} d \sigma<+\infty .
$$

(ii) If $0<q<1$ and $q<r<\infty$, then there exists a positive (super)solution $u \in L^{r}(\Omega, d \sigma)$ to (1.3) if and only if (1.1) holds with $p=\frac{r}{q}$, or equivalently,

$$
\int_{\Omega}(\mathbf{G} \sigma)^{\frac{r}{1-q}} d \sigma<+\infty .
$$

Remark 1.2. We observe that the "if" parts of statements (i) and (ii) of Theorem 1.1 fail if $p=1$, and $r=q$, respectively. The "only if" parts hold for all $0<r<p$ in statement (i), and $r>0$ in statement (ii).

Remark 1.3. It is known that inequality (1.1) with $p=\frac{r}{q} \geq 1$ in the case $0<$ $q<1$ yields the existence of a positive supersolution $u \in L^{r}(\Omega, \sigma)$ for $(1.2)$. This statement follows from a lemma due to Gagliardo [12], and does not require $G$ to be quasi-symmetric or to satisfy the WMP (see Sec. 3 below). However, the converse statement does not hold without the WMP (see [20] in the case $r=q$ ).

Remark 1.4. Without the assumption that $G$ satisfies the WMP, the "only if" parts of statement (i) (with $p=\frac{r}{q} \geq 1$ ) and statement (ii) (with $r \geq q$ ) hold only for $0<r \leq 1-q^{2}$ (see Lemma 3.1 below).

In particular, if there exists a positive (super)solution $u \in L^{q}(\Omega, \sigma)$, then (1.7) holds with $r=q$ for $0<q \leq q_{0}$, where $q_{0}=\frac{\sqrt{5}-1}{2}=0.61 \ldots$ is the conjugate golden ratio. However, (1.7) with $r=q$ generally fails (even for symmetric kernels) in the case $q_{0}<q<1$; the cut-off $q=q_{0}$ here is sharp [20].

In Sec. 4 below, we discuss related results, and provide some counterexamples in the case $p=1$.

\section{KERNELS AND POTENTIAL THEORY}

Let $G: \Omega \times \Omega \rightarrow(0,+\infty]$ be a positive kernel. We will assume that $\Omega$ is a locally compact space Hausdorff space, and $G$ is lower semicontinuous, so that we can apply elements of the classical potential theory developed for such kernels (see [3], [11]). Most of our results hold for non-negative kernels $G(x, y) \geq 0$. In that case, some statements concerning the existence of positive solutions (rather than supersolutions) require the additional assumption that $G$ is non-degenerate; see [20].

By $\mathscr{M}^{+}(\Omega)$ we denote the class of all nonnegative, locally finite, Borel measures on $\Omega$. We use the notation $\operatorname{supp}(v)$ for the support of $v \in \mathscr{M}^{+}(\Omega)$ and $\|v\|=v(\Omega)$ if $v$ is a finite measure.

For $v \in \mathscr{M}^{+}(\Omega)$, the potential of $v$ is defined by

$$
\mathbf{G} v(x):=\int_{\Omega} G(x, y) d v(y), \quad \forall x \in \Omega,
$$


and the potential with the adjoint kernel

$$
\mathbf{G}^{*} v(y):=\int_{\Omega} G(x, y) d v(x), \quad \forall y \in \Omega .
$$

A positive kernel $G$ on $\Omega \times \Omega$ is said to satisfy the weak maximum principle (WMP) with constant $h \geq 1$ if, for any $v \in \mathscr{M}^{+}(\Omega)$,

$$
\sup \{\mathbf{G} v(x): x \in \operatorname{supp}(v)\} \leq M \Longrightarrow \sup \{\mathbf{G} v(x): x \in \Omega\} \leq h M,
$$

for any constant $M>0$. When $h=1, G$ is said to satisfy the strong maximum principle. It holds for Green's kernels associated with the classical Laplacian, or fractional Laplacian $(-\Delta)^{\alpha}$ in the case $0<\alpha \leq 1$, for all domains $\Omega$ with positive Green's function. The WMP holds for Riesz kernels on $\mathbb{R}^{n}$ associated with $(-\Delta)^{\alpha}$ in the full range $0<\alpha<\frac{n}{2}$, and more generally for all radially non-increasing kernels on $\mathbb{R}^{n}$ (see [1]).

The WMP also holds for the so-called quasi-metric kernels (see [8], [9], [15], [20]). We say that $d(x, y): \Omega \times \Omega \rightarrow[0,+\infty)$ satisfies the quasimetric triangle inequality with quasimetric constant $\kappa$ if

$$
d(x, y) \leq \kappa[d(x, z)+d(z, y)],
$$

for any $x, y, z \in \Omega$. We say that $G$ is a quasimetric kernel (with quasimetric constant $\kappa>0)$ if $G$ is symmetric and $d(x, y)=\frac{1}{G(x, y)}$ satisfies (2.2).

A kernel $G: \Omega \times \Omega \rightarrow(0,+\infty]$ is said to be quasi-symmetric if there exists a constant $a$ such that

$$
a^{-1} G(y, x) \leq G(x, y) \leq a G(y, x), \quad \forall x, y \in \Omega .
$$

Many kernels associated with elliptic operators are quasi-symmetric and satisfy the WMP (see [2]).

For $0<q<1$, and $\sigma \in \mathscr{M}^{+}(\Omega)$, we are interested in positive solutions $u \in L^{r}(\sigma)$ $(r>0)$ to the integral equation

$$
u=\mathbf{G}\left(u^{q} \sigma\right), \quad u>0 \quad d \sigma-a . e .
$$

and positive supersolutions $u \in L^{r}(\sigma)$ to the integral inequality

$$
u \geq \mathbf{G}\left(u^{q} \sigma\right), \quad u>0 \quad d \sigma-\text { a.e. }
$$

In [20], we characterized the existence of positive solutions $u \in L^{q}(\Omega, \sigma)$ and $u \in L_{\mathrm{loc}}^{q}(\sigma)$. The latter correspond to the so-called "very weak" solutions to the sublinear boundary value problem (1.4) (see [9], [16]). It is easy to see that the condition $u \in L_{\mathrm{loc}}^{q}(\sigma)$ is necessary for the existence of any positive (super)solution, since otherwise $u \equiv+\infty d \sigma$-a.e. (see [20]).

For a measure $\lambda \in \mathscr{M}^{+}(\Omega)$, the energy of $\lambda$ is given by

$$
\mathscr{E}(\lambda):=\int_{\Omega} \mathbf{G} \lambda d \lambda
$$

The notion of energy is closely related to another major tool of potential theory, the capacity of a set, and the associated equilibrium measure. 
For a kernel $G: \Omega \times \Omega \rightarrow(0,+\infty]$, we consider the Wiener capacity

$$
\operatorname{cap}(K):=\sup \left\{\mu(K): \mathbf{G}^{*} \mu(y) \leq 1 \text { on } \operatorname{supp}(\mu), \mu \in \mathscr{M}^{+}(K)\right\},
$$

defined for compact sets $K \subset \Omega$.

The extremal measure $\mu$ for which the supremum in (2.5) is attained is called the equilibrium measure. Alternatively, capacity can be defined as a solution to the following extremal problem involving energy:

$$
\operatorname{cap}(K):=\left[\inf \left\{\mathscr{E}(\mu): \mu \in \mathscr{M}^{+}(K), \quad \mu(K)=1\right\}\right]^{-1} .
$$

We say that a property holds nearly everywhere (or n.e.) on $K$ when the exceptional set $Z \subset K$ where this property fails has zero capacity, $\operatorname{cap}(Z)=0$.

We will use the following fundamental theorem [3], [11].

Theorem 2.1. Let $G$ be a positive symmetric kernel on $\Omega \times \Omega$, and let $K \subset \Omega$ a compact set. The two extremal problems

$$
\begin{aligned}
& \max \left\{\lambda(K): \mathbf{G} \lambda \leq 1 \text { on } \operatorname{supp}(\lambda), \lambda \in \mathscr{M}^{+}(K)\right\}, \\
& \max \left\{2 \lambda(K)-\mathscr{E}(\lambda): \lambda \in \mathscr{M}^{+}(K)\right\},
\end{aligned}
$$

always have solutions, which are precisely the same, and each maximum coincides with the Wiener capacity cap $K$. The class of all solutions consists of measures $\lambda \in \mathscr{M}^{+}(K)$ for which

$$
\mathscr{E}(\lambda)=\lambda(\Omega)=\operatorname{cap}(K) .
$$

The potential of any solution has the following properties:

(1) $\mathbf{G} \lambda(x) \geq 1$ n.e. in $K$,

(2) $\mathbf{G} \lambda(x) \leq 1$ on $\operatorname{supp}(\lambda)$,

(3) $\mathbf{G} \lambda(x)=1 d \lambda$-a.e. in $\Omega$.

The extremal measure $\lambda$ in Theorem 2.1 is the equilibrium measure for the set $K$. We observe that since $G$ is a positive kernel, the capacity of all compact sets $K$ is finite. (This is true even for non-negative kernels if $G(x, x)>0$ for all $x \in \Omega$; see [11]).

\section{WEIGHTED NORM INEQUALITIES, SUPERSOLUTIONS, AND ENERGY ESTIMATES}

We begin this section with a proof of Theorem 1.1. We remark that the "only if" part of statement (i) of Theorem 1.1 is proved without using the assumption that $G$ is quasi-symmetric. Furthermore, the proof of this part works in the case $p=1$ as well.

Proof of Theorem 1.1. We first prove statement (i). If the $(p, r)$-inequality (1.1) holds for $0<r<p$, where $p \geq 1$, then assuming that $f=(\mathbf{G} \sigma)^{\frac{r}{p-r}} \in L^{p}(\Omega, \sigma)$ and using it as a test function, we deduce

$$
\int_{\Omega}\left[\mathbf{G}\left((\mathbf{G} \sigma)^{\frac{r}{p-r}} d \sigma\right)\right]^{r} d \sigma \leq C^{r}\left[\int_{\Omega}(\mathbf{G} \sigma)^{\frac{p r}{p-r}} d \sigma\right]^{\frac{r}{p}},
$$


where $C$ is the embedding constant in 1.1). We now use the pointwise inequality

$$
[\mathbf{G} \sigma(x)]^{s} \leq s h^{s-1} \mathbf{G}\left((\mathbf{G} \sigma)^{s-1} d \sigma\right)(x), \quad x \in \Omega,
$$

for all $s \geq 1$, established in [14, Lemma 2.5 and Remark 2.6] for non-negative kernels satisfying the WMP with constant $h \geq 1$. Applying (3.1) with $s=\frac{p}{p-r}$, we obtain

$$
\int_{\Omega}(\mathbf{G} \sigma)^{\frac{p r}{p-r}} d \sigma \leq\left(\frac{p}{p-r}\right)^{r} h^{\frac{r^{2}}{p-r}} C^{r}\left[\int_{\Omega}(\mathbf{G} \sigma)^{\frac{p r}{p-r}} d \sigma\right]^{\frac{r}{p}} .
$$

Since $0<r<p$, this estimate yields

$$
\int_{\Omega}(\mathbf{G} \sigma)^{\frac{p r}{p-r}} d \sigma \leq\left(\frac{p}{p-r}\right)^{\frac{p r}{p-r}} h^{\frac{p r^{2}}{(p-r)^{2}}} C^{\frac{p r}{p-r}} .
$$

The extra assumption that $f=(\mathbf{G} \sigma)^{\frac{r}{p-r}} \in L^{p}(\Omega, \sigma)$ is easy to remove by using $\chi_{K} f$ in place of $f$, where $K$ is a compact subset of $\Omega$ on which $\mathbf{G} \sigma(x) \leq n$, and then letting $n \rightarrow+\infty$ (see details in [20]).

In the opposite direction, suppose that (1.6) holds for $0<r<p$ and $p>1$. Without loss of generality we may assume that $f \geq 0$. By Hölder's inequality,

$$
\begin{aligned}
\int_{\Omega}[\mathbf{G}(f d \sigma)]^{r} d \sigma & =\int_{\Omega}\left[\frac{\mathbf{G}(f d \sigma)}{\mathbf{G} \sigma}\right]^{r}(\mathbf{G} \sigma)^{r} d \sigma \\
& \leq\left[\int_{\Omega}\left(\frac{\mathbf{G}(f d \sigma)}{\mathbf{G} \sigma}\right)^{p} d \sigma\right]^{\frac{r}{p}}\left[\int_{\Omega}(\mathbf{G} \sigma)^{\frac{p r}{p-r}} d \sigma\right]^{1-\frac{r}{p}} .
\end{aligned}
$$

We next sketch a proof of a $(1,1)$-weak type estimate obtained in a more general context in [20, Lemma 5.10]:

$$
\left\|\frac{\mathbf{G}(f d \sigma)}{\mathbf{G} \sigma}\right\|_{L^{1, \infty}(\Omega, d \sigma)} \leq c\|f\|_{L^{1}(\Omega, d \sigma)},
$$

where $c=c(h, a)$ depends only on the constants $h \geq 1$ in the weak maximum principle, and $a>0$ in the quasi-symmetry condition.

Since $G$ is quasi-symmetric, we can assume without loss of generality that it is symmetric by replacing $G$ with $\frac{1}{2}\left(G+G^{*}\right)$. Let $E_{t}=\left\{x \in \Omega: \frac{\mathbf{G}(f d \sigma)}{\mathbf{G} \sigma}(x)>t\right\}$, where $t>0$. For an arbitrary compact set $K \subset E_{t}$, we denote by $\mu \in \mathscr{M}^{+}(K)$ an equilibrium measure on $K$ (see Sec. 2 above) such that $\mathbf{G} \mu \geq 1$ n.e. on $K$ and $\mathbf{G} \mu \leq 1$ on $\operatorname{supp}(\mu)$.

It is easy to see that in fact

$$
\mathbf{G} \mu \geq 1 \quad d \sigma-\text { a.e. on } K .
$$

Indeed, from (1.6) it follows that $\mathbf{G} \sigma<+\infty d \sigma$-a.e. Since $\mathbf{G} \mu \geq 1$ n.e. on $K$, the set $Z=\{x \in K: \mathbf{G} \mu(x)<1\}$ has zero capacity, and consequently,

$$
\begin{aligned}
\sigma(Z) & =\sigma(\{x \in Z: \mathbf{G} \sigma(x)<+\infty\}) \\
& \leq \sum_{n=1}^{+\infty} \sigma(\{x \in Z: \mathbf{G} \sigma(x) \leq n\})
\end{aligned}
$$




$$
\leq \sum_{n=1}^{+\infty} n \operatorname{cap}(\{x \in Z: \mathbf{G} \sigma(x) \leq n\})=0 .
$$

Thus, $\sigma(Z)=0$, which proves 3.3 .

Since $\mathbf{G} \mu \leq 1$ on $\operatorname{supp}(\mu)$, it follows that $\mathbf{G} \mu \leq h$ on $\Omega$ by the WMP. From this and (3.3), using Fubini's theorem, we deduce

$$
\begin{aligned}
\sigma(K) & \leq \int_{K} \mathbf{G} \mu d \sigma=\int_{K} \mathbf{G} \sigma_{K} d \mu \\
& \leq \int_{K} \frac{\mathbf{G}(f d \sigma)}{t} d \mu=\frac{1}{t} \int_{K} \mathbf{G} \mu f d \sigma \\
& \leq \frac{1}{t} \int_{\Omega} h f d \sigma=\frac{h}{t}\|f\|_{L^{1}(\Omega, \sigma)} .
\end{aligned}
$$

Taking the supremum over all $K \subset E_{t}$, we obtain

$$
\sigma\left(E_{t}\right) \leq \frac{h}{t}\|f\|_{L^{1}(\Omega, \sigma)},
$$

which proves 3.2 .

The corresponding $L^{\infty}$ estimate is obvious:

$$
\left\|\frac{\mathbf{G}(f d \sigma)}{\mathbf{G} \sigma}\right\|_{L^{\infty}(\Omega, d \sigma)} \leq\|f\|_{L^{\infty}(\Omega, d \sigma)} .
$$

Thus, for $1<p<+\infty$, by the Marcinkiewicz interpolation theorem we obtain

$$
\left\|\frac{\mathbf{G}(f d \sigma)}{\mathbf{G} \sigma}\right\|_{L^{p}(\Omega, d \sigma)} \leq C\|f\|_{L^{p}(\Omega, d \sigma)},
$$

for all $f \in L^{p}(\Omega, d \sigma)$. Hence, combining the preceding estimates, we deduce

$$
\int_{\Omega}[\mathbf{G}(f d \sigma)]^{r} d \sigma \leq C\|f\|_{L^{p}(\Omega, \sigma)}^{r}\left[\int_{\Omega}(\mathbf{G} \sigma)^{\frac{p r}{p-r}} d \sigma\right]^{1-\frac{r}{p}} .
$$

This proves statement (i).

We now prove statement (ii). Let $0<q<1$. Suppose there exists a positive supersolution $u \in L^{r}(\Omega, \sigma)$ with $r>q$. As shown in [14, Corollary 3.6], if $G$ satisfies the WMP, then any nontrivial supersolution $u$ satisfies the global pointwise bound

$$
u(x) \geq(1-q)^{\frac{1}{1-q}} h^{-\frac{q}{1-q}}[\mathbf{G} \sigma(x)]^{\frac{1}{1-q}} \quad d \sigma-\text { a.e. }
$$

Thus, (1.7) holds.

Conversely, by statement (i), 1.7) with $r>q$ implies the $(p, r)$-inequality (1.1) with $p=\frac{r}{q}$. Letting $u_{0}=c[\mathbf{G} \sigma(x)]^{\frac{1}{1-q}}$ where $c>0$ is a positive constant, we get a sequence of iterations

$$
u_{j+1}=\mathbf{G}\left(u_{j}^{q} d \sigma\right), \quad j=0,1, \ldots,
$$

where by induction we see that $u_{j+1} \geq u_{j}$, provided the constant $c$ is small enough. Here the initial step $u_{1} \geq u_{0}$ follows from (3.1) with $s=\frac{1}{1-q}$, since

$$
u_{1}=\mathbf{G}\left(u_{0}^{q} d \sigma\right)=c^{q} \mathbf{G}\left[(\mathbf{G} \sigma)^{\frac{q}{1-q}} d \sigma\right] \geq c[\mathbf{G} \sigma(x)]^{\frac{1}{1-q}}=u_{0},
$$


for an appropriate choice of $c=c(q, h, a)$. By 1.1) with $p=\frac{r}{q}$ and $f=u_{j}$, we have by induction,

$$
\left\|u_{j+1}\right\|_{L^{r}(\Omega, \sigma)}=\left\|\mathbf{G}\left(u_{j}^{q} d \sigma\right)\right\|_{L^{r}(\Omega, \sigma)} \leq C\left\|u_{j}\right\|_{L^{r}(\Omega, \sigma)}^{q}<+\infty .
$$

Since $0<q<1$ and $u_{j} \leq u_{j+1}$, it follows that

$$
\left\|u_{j+1}\right\|_{L^{r}(\Omega, \sigma)} \leq C(r, q, h, a), \quad j=0,1, \ldots .
$$

Using the monotone convergence theorem, we obtain a positive solution

$$
u=\lim _{j \rightarrow \infty} u_{j}, \quad u \in L^{r}(\Omega, \sigma) .
$$

Theorem 1.1 makes use of energy conditions of the type

$$
\int_{\Omega}(\mathbf{G} \sigma)^{s} d \sigma<\infty
$$

for some $s>0$. Note that when $s=1$, this gives the energy $\mathscr{E}(\sigma)$ introduced above.

In the next lemma, we deduce (3.5) for $s=\frac{r}{1-q}$ provided there exists a positive supersolution $u \in L^{r}(\Omega, \sigma)$ to 1.2 , for non-negative, quasi-symmetric kenels $G$, without assuming that 1.1 holds, or that $G$ satisfies the WMP. In the special case $r=q$ it was proved in [20, Lemma 5.1].

Lemma 3.1. Let $\sigma \in \mathscr{M}^{+}(\Omega)$, and let $0<q<1$. Suppose $G$ is a non-negative quasi-symmetric kernel on $\Omega \times \Omega$. Suppose there is a positive supersolution $u \in$ $L^{r}(\Omega, \sigma)(r>0)$, i.e., $\mathbf{G}\left(u^{q} d \sigma\right) \leq u d \sigma$-a.e. Let $0<q \leq 1-r^{2}$. Then

$$
\int_{\Omega}(\mathbf{G} \sigma)^{\frac{r}{1-q}} d \sigma \leq a^{\frac{r q}{(1-q)(1-r+q)}} \int_{\Omega} u^{r} d \sigma<+\infty
$$

where $a$ is the quasi-symmetry constant of $G$.

Proof. Suppose $u \in L^{r}(\Omega, \sigma)$, where $0<r<1$, is a positive supersolution. Let $\gamma \geq 1$. By Hölder's inequality with exponents $\gamma$ and $\gamma^{\prime}=\frac{\gamma}{\gamma-1}$, we estimate

$$
\begin{aligned}
\mathbf{G} \sigma(x) & =\int_{\Omega} u^{\frac{q}{\gamma}} u^{-\frac{q}{\gamma}} G(x, y) d \sigma(y) \\
& \leq\left[\mathbf{G}\left(u^{q} d \sigma(x)\right]^{\frac{1}{\gamma}}\left[\mathbf{G}\left(u^{-\frac{q}{\gamma-1}} d \sigma\right)(x)\right]^{\frac{1}{\gamma}}\right. \\
& \leq[u(x)]^{\frac{1}{\gamma}}\left[\mathbf{G}\left(u^{-\frac{q}{\gamma-1}} d \sigma\right)(x)\right]^{\frac{1}{\gamma}} .
\end{aligned}
$$

Let $\gamma=1+\frac{q}{1-r}$, where $0<r \leq 1-q^{2}$. Then $\frac{(1-q) \gamma^{\prime}}{r} \geq 1$. Using the preceding inequality, along with Hölder's inequality with the conjugate exponents

$$
\frac{(1-q)(1-r+q)}{1-r-q^{2}}>1 \text { and } \quad \frac{(1-q)(1-r+q)}{r q} \geq 1,
$$

and Fubini's theorem, we estimate 


$$
\begin{aligned}
\int_{\Omega}(\mathbf{G} \sigma)^{\frac{r}{1-q}} d \sigma & \leq \int_{\Omega} u^{\frac{r}{(1-q) \gamma}}\left[\mathbf{G}\left(u^{r-1} d \sigma\right)\right]^{\frac{r}{(1-q) \gamma}} d \sigma \\
& =\int_{\Omega} u^{\frac{r\left(1-r-q^{2}\right)}{(1-q)(1-r+q)}}\left[u^{q} \mathbf{G}\left(u^{r-1} d \sigma\right)\right]^{\frac{r q}{(1-q)(1-r+q)}} d \sigma \\
& \leq\left[\int_{\Omega} u^{r} d \sigma\right]^{\frac{1-r-q^{2}}{(1-q)(1-r+q)}}\left[\int_{\Omega} \mathbf{G}\left(u^{r-1} d \sigma\right) u^{q} d \sigma\right]^{\frac{r q}{(1-q)(1-r+q)}} \\
& =\left[\int_{\Omega} u^{r} d \sigma\right]^{\frac{1-r-q^{2}}{(1-q)(1-r+q)}}\left[\int_{\Omega} \mathbf{G}^{*}\left(u^{q} d \sigma\right) u^{r-1} d \sigma\right]^{\frac{r q}{(1-q)(1-r+q)}} \\
& \leq a^{\frac{r q}{(1-q)(1-r+q)}}\left[\int_{\Omega} u^{r} d \sigma\right]^{\frac{1-r-q^{2}+r q}{(1-q)(1-r+q)}} .
\end{aligned}
$$

In the last estimate we used the inequality $\mathbf{G}^{*}\left(u^{q} d \sigma\right) \leq a u$. Since $1-r-q^{2}+r q=$ $(1-q)(1-r+q)$, this completes the proof of (3.6).

We next show that, for general non-negative kernels $G$, the $(p, r)$-weighted norm inequality (1.1) with $p=\frac{r}{q} \geq 1$ yields the existence of a supersolution $u \in L^{r}(\Omega, \sigma)$ to (1.2). This is deduced from Gagliardo's lemma [12] (see also [22]), as in the special case $r=q$ in [20].

It will be convenient for us to construct a measurable function $\phi$ such that

$$
0<[\mathbf{G}(\phi d \sigma)]^{q} \leq \phi<+\infty \quad d \sigma-\text { a.e. }
$$

for $0<q<1$. Clearly, if $\phi$ satisfies the above estimate, then $u=\phi^{\frac{1}{q}}$ satisfies (1.2). Moreover, $u \in L^{r}(\Omega, \sigma)$ if $\phi \in L^{p}(\Omega, \sigma)$, where $p=\frac{r}{q} \geq 1$.

We recall that a convex cone $P \subset B$ is strictly convex at the origin if, for any $\phi, \psi \in P, \alpha \phi+\beta \psi=0$ implies $\phi=\psi=0$, for any $\alpha, \beta>0$ such that $\alpha+\beta=1$.

Lemma 3.2 (Gagliardo [12]). Let $B$ be a Banach space, and let $P \subset B$ be a convex cone which is strictly convex at the origin and such that if $\left(\phi_{n}\right) \subset P, \phi_{n+1}-\phi_{n} \in P$, and $\left\|\phi_{n}\right\| \leq M$ for all $n=1,2, \ldots$, then there exists $\phi \in P$ so that $\left\|\phi_{n}-\phi\right\| \rightarrow 0$.

Let $S: P \rightarrow P$ be a continuous mapping with the following properties:

(1) For $\phi, \psi \in P$, such that $\phi-\psi \in P$, we have $S \phi-S \psi \in P$.

(2) If $\|\phi\| \leq 1$ and $\phi \in P$, then $\|S u\| \leq 1$.

Then for every $\lambda>0$ there exists $\phi \in P$ so that $(1+\lambda) \phi-S \phi \in P$ and $0<\|\phi\| \leq 1$. Moreover, for every $\psi \in P$ such that $0<\|\psi\|_{B} \leq \frac{\lambda}{1+\lambda}$, $\phi$ can be chosen so that $\phi=\psi+\frac{1}{1+\lambda} S \phi$.

We will apply this lemma to $B=L^{p}(\sigma), p \geq 1$, and the cone of non-negative functions $P$ in $B$. In this case obviously one can ensure that $\phi>0 d \sigma$-a.e.

Lemma 3.3. Let $(\Omega, \sigma)$ be a sigma-finite measure space, and let $G$ be a nonnegative kernel on $\Omega \times \Omega$. Let $0<r<+\infty$ and $0<q<1$. Suppose 1.1) holds for 
$p=\frac{r}{q} \geq 1$ with an embedding constant $C=\varkappa>0$. Then, for every $\lambda>0$, there is a positive $\phi \in L^{p}(\sigma)$ satisfying (3.7) so that

$$
\|\phi\|_{L^{p}(\sigma)} \leq(1+\lambda)^{\frac{1}{1-q}} \varkappa^{\frac{q}{1-q}} .
$$

Proof. The supersolution $\phi$ is constructed using Lemma 3.2. Define $S: L^{p}(\sigma) \rightarrow$ $L^{p}(\sigma)$ by

$$
S \phi:=\left[\frac{1}{\varkappa^{q}} \mathbf{G}(\phi d \sigma)\right]^{q},
$$

for all $\phi \in L^{p}(\sigma), \phi \geq 0$. Inequality (1.1) gives that $S$ is a bounded continuous operator. In fact, by (1.1) we see that if $\|\phi\|_{L^{p}(\sigma)} \leq 1$, then

$$
\begin{aligned}
\|S(\phi)\|_{L^{p}(\sigma)}^{p} & =\frac{1}{\varkappa^{r}} \int_{\Omega}[\mathbf{G}(\phi \sigma)]^{r} d \sigma \\
& =\frac{1}{\varkappa^{r}} \varkappa^{r}\left(\int_{\Omega} \phi^{p} d \sigma\right)^{q} \leq 1 .
\end{aligned}
$$

Therefore, by Lemma 3.2, there exists $\phi \in L^{p}(\sigma)$ such that

$$
(1+\lambda) \phi \geq \frac{1}{\varkappa^{q}}[\mathbf{G}(\phi \sigma)]^{q},
$$

$\|\phi\|_{L^{p}(\sigma)} \leq 1$, and $\phi>0 d \sigma$-a.e. Setting $\phi_{0}=c \phi$, where

$$
c=\left[\frac{1}{(1+\lambda) \varkappa^{q}}\right]^{\frac{1}{1-q}},
$$

we deduce that $\phi>0 d \sigma$-a.e., and

$$
\phi_{0} \geq \mathbf{G}\left(\phi_{0} \sigma\right)^{q}, \quad\left\|\phi_{0}\right\|_{L^{p}(\sigma)} \leq(1+\lambda)^{\frac{1}{1-q}} \varkappa^{\frac{q}{1-q}} .
$$

Remark 1.3 follows immediately from Lemma 3.3 .

Remark 3.4. For $p=\frac{r}{q}$, a counterexample in [20] demonstrates that, without the WMP, the existence of a supersolution $u \in L^{r}(\Omega, \sigma)$ to (1.2) in the case $r=q$ does not imply the $(p, r)$-weighted norm inequality (1.1), even for positive symmetric kernels $G$. A slight modification of that counterexample shows that the same is true in the case $r>q$ as well.

\section{A COUNTEREXAMPLE IN THE END-POINT CASE $p=1$}

In the case $p=1,0<q<1$, the $(1, q)$-weighted norm inequality (1.1) with $r=q$ follows from a similar inequality for the space of measures $\mathscr{M}^{+}(\Omega)$ in place of $L^{1}(\Omega, \sigma)$,

$$
\|\mathbf{G} v\|_{L^{q}(\Omega, \sigma)} \leq C\|v\|, \quad \forall v \in \mathscr{M}^{+}(\Omega),
$$

where $\|v\|=v(\Omega)$. This inequality was shown in [20] to be equivalent to the existence of a positive supersolution $u \in L^{q}(\Omega, \sigma)$ to (1.2) for quasi-symmetric kernels $G$ satisfying the WMP. In this case, 4.1) is equivalent to (1.1) with $r=q$ and $p=1$ in view of Lemma 3.3 . 
However, a characterization of (4.1), or (1.1) with $r=q$ and $p=1$, in terms of the energy estimate 1.7) with $r=q$ is not available, contrary to the case $r>q$ : the condition

$$
\int_{\Omega}(\mathbf{G} \sigma)^{\frac{q}{1-q}} d \sigma<+\infty
$$

is not sufficient for (4.1).

On the other hand, it is not difficult to see that 4.1) holds for all $v \in \mathscr{M}^{+}(\Omega)$ if and only if it holds for all finite linear combinations of point masses, $v=$ $\sum_{j=1}^{n} a_{j} \delta_{x_{j}}, a_{j}>0$. It had been conjectured that, for $0<q<1$, condition (4.2) combined with (4.1) for single point masses $v=\delta_{x}$, i.e.,

$$
\int_{\Omega} G(x, y)^{q} d \sigma(y) \leq C<+\infty, \quad \forall x \in \Omega,
$$

was not only necessary, but also sufficient for 4.1). (Notice that in the case $q \geq 1$ (4.3) is obviously necessary and sufficient for [4.1); see [20].)

In this section, we give a counterexample to this conjecture for Riesz potentials on $\mathbb{R}^{n}$,

$$
\mathbf{I}_{2 \alpha} v(x)=\int_{\mathbb{R}^{n}} \frac{d v(y)}{|x-y|^{n-2 \alpha}}, \quad x \in \mathbb{R}^{n},
$$

where $v \in \mathscr{M}^{+}\left(\mathbb{R}^{n}\right)$, and $0<2 \alpha<n$. Clearly, Riesz kernels $|x-y|^{2 \alpha-n}$ are symmetric, and satisfy the WMP.

Suppose $0<q<1, n \geq 1$, and $0<2 \alpha<n$. We construct $\sigma \in \mathscr{M}^{+}\left(\mathbb{R}^{n}\right)$ such that

$$
\mathscr{E}(\sigma)=\mathscr{E}_{\alpha, q}(\sigma):=\int_{\mathbb{R}^{n}}\left(\mathbf{I}_{2 \alpha} \sigma\right)^{\frac{q}{1-q}} d \sigma<+\infty
$$

and

$$
\mathscr{K}(\sigma)=\mathscr{K}_{\alpha, q}(\sigma):=\sup _{x \in \mathbb{R}^{n}} \int_{\mathbb{R}^{n}} \frac{d \sigma(y)}{|x-y|^{(n-2 \alpha) q}}<+\infty,
$$

but

$$
\kappa(\sigma)=\kappa(\sigma)_{\alpha, q}:=\sup \left\{\frac{\left\|\mathbf{I}_{2 \alpha} v\right\|_{L^{q}(\sigma)}}{\|v\|_{\mathscr{M}^{+}\left(\mathbb{R}^{n}\right)}}: v \in \mathscr{M}^{+}\left(\mathbb{R}^{n}\right), v \neq 0\right\}=+\infty .
$$

In other words, we need to construct a measure $\sigma$ such that $\mathscr{E}(\sigma)<+\infty$ (in the special case $q=\frac{1}{2}$ this means that $\sigma$ has finite energy), and (4.6) holds for all $\delta$-functions $v=\delta_{x}\left(x \in \mathbb{R}^{n}\right)$, but (4.6) fails for a linear combination of $\delta$-functions

$$
v=\sum_{j=1}^{\infty} a_{j} \delta_{x_{j}}, \quad \text { where } \quad \sum_{j=1}^{\infty} a_{j}<+\infty, \quad a_{j}>0 .
$$

We will use a modification of the example considered in [6] for other purposes.

We will need the following lemma and its corollary in the radially symmetric case (see [6]). 
Lemma 4.1. Let $0<q<1$ and $0<2 \alpha<n$. If $d \sigma=\sigma(|x|) d x$ is radially symmetric, then $\kappa(\sigma)<+\infty$ if and only if $\mathscr{K}(\sigma)<+\infty$. Moreover, there exists a constant $c=c(q, \alpha, n)>0$ such that $\kappa(\sigma)$ satisfies

$$
\mathscr{K}(\sigma) \leq \kappa(\sigma)^{q} \leq c \mathscr{K}(\sigma),
$$

where in the this case

$$
\mathscr{K}(\sigma)=\int_{\mathbb{R}^{n}} \frac{d \sigma(y)}{|y|^{(n-2 \alpha) q}} .
$$

Remark 4.2. For radially symmetric $\sigma$, condition $\mathscr{K}(\sigma)<+\infty$ is equivalent to $\sigma \in L^{\frac{1}{1-q}, 1}\left(\mathbb{R}^{n}, \sigma\right)$, which is necessary and sufficient for (4.1) in this case; see [19], [20]. Here $L^{s, 1}\left(\mathbb{R}^{n}, \sigma\right)$ denotes the corresponding Lorentz space with respect to the measure $\sigma$.

Corollary 4.3. Let $\sigma_{R, \gamma}=\chi_{B(0, R)}|x|^{-\gamma}$, where $0 \leq \gamma<n-q(n-2 \alpha)$ and $R>0$. Then

$$
\mathscr{K}(\sigma)=\frac{\omega_{n} R^{n-\gamma-q(n-2 \alpha)}}{n-\gamma-q(n-2 \alpha)}
$$

and

$$
\frac{\omega_{n}}{n-\gamma-q(n-2 \alpha)} \leq \frac{\kappa\left(\sigma_{R, \gamma}\right)^{q}}{R^{n-\gamma-q(n-2 \alpha)}} \leq \frac{c}{n-\gamma-q(n-2 \alpha)},
$$

where $c=c(q, \alpha, n)$, and $\omega_{n}=\left|S^{n-1}\right|$ is the surface area of the unit sphere.

Let

$$
\sigma=\sum_{k=1}^{\infty} c_{k} \sigma_{k}
$$

where

$$
\sigma_{k}=\sigma_{R_{k}, \gamma_{k}}\left(x+x_{k}\right), \quad R_{k}=\left|x_{k}\right|=k, \quad \gamma_{k}=n-q(n-2 \alpha)-\varepsilon_{k},
$$

and the positive scalars $c_{k}, \varepsilon_{k}$ are picked so that $\sum_{k=1}^{\infty} c_{k}<\infty, \varepsilon_{k} \rightarrow 0$, and $0<\gamma_{k}<n$. Notice that $\gamma_{k} \rightarrow n-q(n-2 \alpha)$ as $k \rightarrow \infty$, which is a critical exponent for the inequality 4.17) (with $\sigma_{k}$ in place of $\sigma$ ) discussed below.

More precisely, for $0<q<1$ and $0<\delta<+\infty$, we set

$$
a_{k}=\frac{1}{k(\log (k+1))^{\frac{1}{q}}}, \quad c_{k}=\frac{1}{k^{2-q+\delta}}, \quad \varepsilon_{k}=\frac{1}{k^{1+\delta}}, \quad k=1,2, \ldots,
$$

so that

$$
\sum_{k=1}^{+\infty} a_{k}<+\infty, \quad \sup _{k \geq 1} \frac{c_{k}}{\varepsilon_{k}}<+\infty, \quad \sum_{k=1}^{+\infty} \frac{c_{k}}{\varepsilon_{k}^{1-q}}<+\infty, \quad \text { but } \quad \sum_{k=1}^{+\infty} \frac{c_{k} a_{k}^{q}}{\varepsilon_{k}}=+\infty .
$$

We first verify condition (4.4). Notice that

$$
c_{1} A \leq\left[\mathscr{E}_{\alpha, q}(\sigma)\right]^{1-q} \leq c_{2} A,
$$


where $A$ is the least constant in the inequality (see [4]; [5], Lemma 3.3)

$$
\int_{\mathbb{R}^{n}}\left|\mathbf{I}_{\alpha} f\right|^{1+q} d \sigma \leq A\|f\|_{L^{2}(d x)}^{1+q}, \quad \text { for all } f \in L^{2}\left(\mathbb{R}^{n}, d x\right),
$$

or, equivalently,

$$
\int_{\mathbb{R}^{n}}\left|\mathbf{I}_{2 \alpha}(g d \sigma)\right|^{1+q} d \sigma \leq A^{2}\|g\|_{L^{\frac{1+q}{q}}(d \sigma)}^{1+q}, \quad \text { for all } g \in L^{2}\left(\mathbb{R}^{n}, \sigma\right),
$$

where the constants of equivalence $c_{1}, c_{2}$ in (4.16) depend only on $\alpha, q$, and $n$.

Consequently, $\left[\mathscr{E}_{\alpha, q}(\sigma)\right]^{1-q}$ is equivalent to a norm on a subset of $\mathscr{M}^{+}\left(\mathbb{R}^{n}\right)$, so that

$$
\left[\mathscr{E}_{\alpha, q}\left(\sum_{k} \sigma_{k}\right)\right]^{1-q} \leq c \sum_{k}\left[\mathscr{E}_{\alpha, q}\left(\sigma_{k}\right)\right]^{1-q},
$$

where $c=c(\alpha, q, n)$ is a positive constant which depends only on $\alpha, q$, and $n$.

We claim that,

$$
\mathscr{E}_{\alpha, q}\left(\sigma_{k}\right) \leq \frac{C R_{k}^{\frac{\varepsilon_{k}}{1-q}}}{\varepsilon_{k}}, \quad k=1,2, \ldots
$$

where $C=C(\alpha, q, n)$.

Indeed, by the semigroup property of Riesz kernels,

$$
\begin{aligned}
\mathbf{I}_{2 \alpha} \sigma_{k}(x) & =c(\alpha, n) \int_{B\left(0, R_{k}\right)} \frac{d t}{|x-t|^{n-2 \alpha}\left|t+x_{k}\right|^{\gamma_{k}}} \\
& \leq c(\alpha, n) \int_{\mathbb{R}^{n}} \frac{d t}{|x-t|^{n-2 \alpha}\left|t+x_{k}\right| \gamma_{k}}=c\left|x+x_{k}\right|^{2 \alpha-\gamma_{k}},
\end{aligned}
$$

where $c=c\left(n, 2 \alpha+n-\gamma_{k}\right)$ remains bounded by a constant $C(\alpha, q, n)$ as $k \rightarrow+\infty$, since $\lim _{k \rightarrow+\infty}\left(2 \alpha+n-\gamma_{k}\right)=2 \alpha+q(n-2 \alpha)<n$.

Notice that $\left(\gamma_{k}-2 \alpha\right) \frac{q}{1-q}+\gamma_{k}=n-\frac{\varepsilon_{k}}{1-q}$. Hence, by the preceding estimate,

$$
\begin{aligned}
\mathscr{E}_{\alpha, q}\left(\sigma_{k}\right) & =\int_{\mathbb{R}^{n}}\left(\mathbf{I}_{2 \alpha} \sigma_{k}\right)^{\frac{q}{1-q}} d \sigma_{k} \\
& \leq c^{\frac{q}{1-q}} \int_{\left|x+x_{k}\right|<R_{k}} \frac{d x}{\left|x+x_{k}\right|^{n-\frac{\varepsilon_{k}}{1-q}}} \\
& =c^{\frac{q}{1-q}} \omega_{n} \int_{0}^{R_{k}} r^{\frac{\varepsilon_{k}}{1-q}-1} d r \\
& \leq \frac{C(\alpha, q, n) R_{k}{ }^{\frac{\varepsilon_{k}}{1-q}}}{\varepsilon_{k}}
\end{aligned}
$$

which proves 4.20).

It follows from (4.19) and the preceding estimate that, for $\sigma$ defined by (4.12),

$$
\begin{aligned}
{\left[\mathscr{E}_{\alpha, q}(\sigma)\right]^{1-q} } & \leq c(\alpha, q, n) \sum_{k} c_{k}\left[\mathscr{E}_{\alpha, q}\left(\sigma_{k}\right)\right]^{1-q} \\
& \leq c(\alpha, q, n) C(\alpha, q, n)^{1-q} \sum_{k} \frac{c_{k} R_{k}^{\varepsilon_{k}}}{\varepsilon_{k}^{1-q}}<+\infty
\end{aligned}
$$


by (4.15), since obviously $\sup _{k>1} R_{k}^{\varepsilon_{k}}<+\infty$ by (4.14). This proves (4.4).

To prove 4.5], we will need the following lemma.

Lemma 4.4. Let $R>0,0<\beta<n$, and $0<\varepsilon<n-\beta$. For $\gamma=n-\beta-\varepsilon>0$, we have

$$
\phi_{R, \gamma}(x):=\int_{|t|<R} \frac{d t}{|x-t|^{\beta}|t|^{\gamma}} \approx \begin{cases}\frac{R^{\varepsilon}-|x|^{\varepsilon}}{\varepsilon} & \text { if }|x| \leq \frac{R}{2}, \\ R^{\varepsilon}\left(\frac{R}{|x|}\right)^{\beta} & \text { if }|x|>\frac{R}{2},\end{cases}
$$

where the constants of equivalence depend only on $\beta$ and $n$.

Proof. Suppose first that $|x|>\frac{R}{2}$. Then

$$
\begin{aligned}
\phi_{R, \gamma}(x) & =\int_{|t|<\frac{R}{4}} \frac{d t}{|x-t|^{\beta}|t|^{\gamma}}+\int_{\frac{R}{4}<|t|<R} \frac{d t}{|x-t|^{\beta}|t|^{\gamma}} \\
& :=I+I I .
\end{aligned}
$$

Clearly, in the first integral $\frac{|x|}{2} \leq|x-t| \leq \frac{3|x|}{2}$, and so $I$ is bounded above and below by

$$
\frac{\omega_{n} c(\beta)}{|x|^{\beta}} \int_{0}^{R} r^{n-1-\gamma} d r=\frac{c(\beta, n) R^{n-\gamma}}{|x|^{\beta}} .
$$

To estimate the second term, notice that, for $|x|>2 R$ and $|t|<R$, we have $|x-t|>$ $\frac{|x|}{2}$, so that

$$
I I \leq \frac{c(\beta, n)}{R^{\gamma}|x|^{\beta}} \int_{\frac{R}{4}<|t|<R} d t=\frac{c(\beta, n) R^{n-\gamma}}{|x|^{\beta}} .
$$

For $\frac{R}{2}<|x|<2 R$ and $|t|<R$, we have $|x-t|<3 R$, and consequently

$$
\begin{aligned}
I I & \leq \frac{c(\beta, n)}{R^{\gamma}} \int_{|x-t|<3 R} \frac{d t}{|x-t|^{\beta}} \\
& =\frac{\omega_{n} c(\beta, n)}{R^{\gamma}} \int_{0}^{3 R} r^{n-1-\beta} d r \\
& =C(\beta, n) R^{n-\beta-\gamma} \\
& \leq \frac{C(\beta, n) R^{n-\gamma}}{|x|^{\beta}} .
\end{aligned}
$$

Thus, $I I \leq c(n, \beta) I$, which proves (4.22) in the case $|x| \geq \frac{R}{2}$.

Suppose now that $|x| \leq \frac{R}{2}$. Then

$$
\begin{aligned}
\phi_{R, \gamma}(x) & =\int_{|t|<\frac{|x|}{2}} \frac{d t}{|x-t|^{\beta}|t|^{\gamma}}+\int_{\frac{|x|}{2}<|t|<2|x|} \frac{d t}{|x-t|^{\beta}|t|^{\gamma}}+\int_{2|x|<|t|<R} \frac{d t}{|x-t|^{\beta}|t|^{\gamma}} \\
& :=I I I+I V+V .
\end{aligned}
$$

Clearly, in the first integral $\frac{|x|}{2}<|x-t|<\frac{3|x|}{2}$, and so III is bounded above and below by

$$
\frac{c(\beta)}{|x|^{\beta}} \int_{|t|<\frac{|x|}{2}} \frac{d t}{|t|^{\gamma}}=\frac{\omega_{n} c(\beta)}{|x|^{\beta}} \int_{0}^{\frac{|x|}{2}} r^{n-1-\gamma} d r=\frac{c(\beta, n)}{(n-\gamma) 2^{n-\gamma}}|x|^{\varepsilon} .
$$


The second integral $I V$ is bounded above and below by

$$
\frac{c(\gamma)}{|x|^{\gamma}} \int_{\frac{|x|}{2}<|t|<2|x|} \frac{d t}{|x-t|^{\beta}}
$$

Clearly,

$$
\begin{aligned}
I V & \leq \frac{c(\gamma)}{|x|^{\gamma}} \int_{|x-t|<3|x|} \frac{d t}{|x-t|^{\beta}} \\
& =\frac{\omega_{n} c(\gamma)}{|x|^{\gamma}} \int_{0}^{3|x|} r^{n-1-\beta} d r \\
& =\frac{\omega_{n} c(\gamma)}{|x|^{\beta+\gamma-n}}=c(\beta, \gamma, n)|x|^{\varepsilon},
\end{aligned}
$$

so that $I V \leq c(\beta, n) I I I$.

Finally, the integral $V$ is bounded above and below by

$$
\begin{aligned}
& c(\beta) \int_{2|x|<|t|<R} \frac{d t}{|t|^{\gamma+\beta}} \\
& =c(\beta) \int_{2|x|}^{R} r^{n-1-\gamma-\beta} d r \\
& =c(\beta) \frac{R^{\varepsilon}-(2|x|)^{\varepsilon}}{\varepsilon} .
\end{aligned}
$$

Combining these estimates we complete the proof of (4.22).

By Lemma 4.4 with $\beta=(n-2 \alpha) q, R=R_{k}, \varepsilon=\varepsilon_{k}$, and $\gamma=\gamma_{k}=n-\beta-\varepsilon_{k}$, we obtain, for $k=2,3, \ldots$,

$$
\begin{aligned}
\phi_{R_{k}, \gamma_{k}}\left(x-x_{k}\right) & =\int_{\left|t+x_{k}\right|<R_{k}} \frac{d t}{|x-t|^{(n-2 \alpha) q}\left|t+x_{k}\right|^{\gamma}} \\
& \leq C(\alpha, q, n) \begin{cases}\frac{R_{k}^{\varepsilon_{k}}}{\varepsilon_{k}} & \text { if }\left|x-x_{k}\right|<1, \\
\frac{R_{k}^{\varepsilon_{k}}-1}{\varepsilon_{k}} & \text { if } 1 \leq\left|x-x_{k}\right| \leq \frac{R_{k}}{2}, \\
R_{k}^{\varepsilon_{k}} & \text { if }\left|x-x_{k}\right|>\frac{R_{k}}{2} .\end{cases}
\end{aligned}
$$

In the case $k=1$, we use the estimate $\phi_{R_{1}, \gamma_{1}}\left(x-x_{1}\right) \leq C(\alpha, q, n) \frac{R_{1}^{\varepsilon_{1}}}{\varepsilon_{1}}$ for all $x \in \mathbb{R}^{n}$. 
We next estimate

$$
\begin{aligned}
\mathscr{K}(\sigma) & =\sup _{x \in \mathbb{R}^{n}} \sum_{k=1}^{+\infty} c_{k} \phi_{R_{k}, \gamma_{k}}\left(x-x_{k}\right) \\
& \leq \sup _{x \in \mathbb{R}^{n}} \sum_{\left|x-x_{k}\right| \leq 1} c_{k} \phi_{R_{k}, \gamma_{k}}\left(x-x_{k}\right) \\
& +\sup _{x \in \mathbb{R}^{n}} \sum_{1<\left|x-x_{k}\right|<\frac{R_{k}}{2}} c_{k} \phi_{R_{k}, \gamma_{k}}\left(x-x_{k}\right) \\
& +\sup _{x \in \mathbb{R}^{n}} \sum_{\left|x-x_{k}\right| \geq \frac{R_{k}}{2}} c_{k} \phi_{R_{k}, \gamma_{k}}\left(x-x_{k}\right) \\
& :=I+I I+I I I .
\end{aligned}
$$

Suppose that $j \leq|x| \leq j+1$ for some $j=0,1, \ldots$. We first estimate $I$. Since $\left|x-x_{k}\right| \leq 1$, and $\left|x_{k}\right|=k$, it follows that

$$
k=\left|x_{k}\right| \leq 1+|x| \leq 1+\left|x-x_{k}\right|+\left|x_{k}\right|=k+2 .
$$

Consequently, $j-1 \leq k \leq j+2$ if $j \geq 2$, and $1 \leq k \leq 3$ if $j=0,1,2$. Hence, the corresponding sum contains no more than four terms, and therefore

$$
\begin{aligned}
I & :=\sup _{x \in \mathbb{R}^{n}} \sum_{\left|x-x_{k}\right| \leq 1} c_{k} \phi_{R_{k}, \gamma_{k}}\left(x-x_{k}\right) \\
& \leq C(\alpha, q, n) \sup _{j \geq 0} \sum_{\max (j-1,1) \leq k \leq \max (j+2,3)} \frac{c_{k} R_{k}^{\varepsilon_{k}}}{\varepsilon_{k}} \\
& \leq C(\alpha, q, n),
\end{aligned}
$$

since by (4.13) and 4.15),

$$
\sup _{k \geq 1} R_{k}^{\varepsilon_{k}}<+\infty, \quad \text { and } \quad \sup _{k \geq 1} \frac{c_{k}}{\varepsilon_{k}}<+\infty .
$$

To estimate $I I$, notice that $0<\varepsilon_{k} \log R_{k} \leq C$, and consequently

$$
\frac{R_{k}^{\varepsilon_{k}}-1}{\varepsilon_{k}} \leq C \log R_{k}
$$

Hence, by (4.23) and (4.14),

$$
\begin{aligned}
I I & :=\sup _{x \in \mathbb{R}^{n}} \sum_{1<\left|x-x_{k}\right|<\frac{R_{k}}{2}} c_{k} \phi_{R_{k}, \gamma_{k}}\left(x-x_{k}\right) \\
& \leq C(\alpha, q, n) \sup _{x \in \mathbb{R}^{n}} \sum_{1<\left|x-x_{k}\right|<\frac{R_{k}}{2}} \frac{c_{k}\left(R_{k}^{\varepsilon_{k}}-1\right)}{\varepsilon_{k}} \\
& \leq C(\alpha, q, n) \sum_{k=1}^{+\infty} c_{k} \log R_{k}<+\infty .
\end{aligned}
$$


Finally, we estimate III using (4.23) and (4.14). Since $\sup _{k} R_{k}^{\varepsilon_{k}}<+\infty$, we deduce

$$
\begin{aligned}
I I I & :=\sup _{x \in \mathbb{R}^{n}} \sum_{\left|x-x_{k}\right| \geq \frac{R_{k}}{2}} c_{k} \phi_{R_{k}, \gamma_{k}}\left(x-x_{k}\right) \\
& \leq C(\alpha, q, n) \sup _{x \in \mathbb{R}^{n}} \sum_{\left|x-x_{k}\right| \geq \frac{R_{k}}{2}} c_{k} R_{k}^{\varepsilon_{k}} \\
& \leq C(\alpha, q, n) \sum_{k=1}^{+\infty} c_{k} \leq C(\alpha, q, n) .
\end{aligned}
$$

This proves 4.5).

It remains to verify (4.6) for $\sigma=\sum_{k=1}^{+\infty} c_{k} \sigma_{k}$ and $v=\sum_{j=1}^{+\infty} a_{j} \delta_{x_{j}}$ defined above. We estimate

$$
\begin{aligned}
\left\|\mathbf{I}_{2 \alpha} v\right\|_{L^{q}(\sigma)}^{q} & =\sum_{k=1}^{+\infty} c_{k} \int_{\mathbb{R}^{n}}\left(\sum_{j=1}^{+\infty} \frac{a_{j}}{\left|x+x_{j}\right|^{n-2 \alpha}}\right)^{q} d \sigma_{k} \\
& \geq \sum_{k=1}^{+\infty} c_{k} \int_{\mathbb{R}^{n}} \frac{a_{k}^{q}}{\left|x+x_{k}\right|^{q(n-2 \alpha)}} d \sigma_{k} \\
& =\sum_{k=1}^{+\infty} c_{k} a_{k}^{q} \int_{\left|x+x_{k}\right|<R_{k}} \frac{d x}{\left|x+x_{k}\right|^{(n-2 \alpha) q+\gamma_{k}}} .
\end{aligned}
$$

Since

$$
\begin{aligned}
\int_{\left|x+x_{k}\right|<R_{k}} \frac{d x}{\left|x+x_{k}\right|^{(n-2 \alpha) q+\gamma_{k}}} & =\int_{|x|<R_{k}} \frac{d x}{|x|^{(n-2 \alpha) q+\gamma_{k}}} \\
& =\omega_{n} \int_{0}^{R_{k}} r^{-1+\varepsilon_{k}} d r=\omega_{n} \frac{R_{k}^{\varepsilon_{k}}}{\varepsilon_{k}},
\end{aligned}
$$

and $R_{k}^{\varepsilon_{k}} \geq 1$, it follows by (4.14) that

$$
\begin{aligned}
\left\|\mathbf{I}_{2 \alpha} v\right\|_{L^{q}(\boldsymbol{\sigma})}^{q} & \geq \omega_{n} \sum_{k=1}^{+\infty} \frac{c_{k} a_{k}^{q}}{\varepsilon_{k}} \\
& =\omega_{n} \sum_{k=1}^{+\infty} \frac{1}{k \log (k+1)}=+\infty
\end{aligned}
$$

\section{REFERENCES}

[1] D. R. Adams and L. I. Hedberg, Function Spaces and Potential Theory, Grundlehren der math. Wissenschaften 314, Berlin-Heidelberg-New York, Springer, 1996.

[2] A. Ancona, Some results and examples about the behaviour of harmonic functions and Green's functions with respect to second order elliptic operators, Nagoya Math. J. 165 (2002), 123-158.

[3] M. Brelot, Lectures on Potential Theory, Lectures on Math. 19, Tata Institute, Bombay, 1960.

[4] C. Cascante, J. M. Ortega, and I. E. Verbitsky, On $L^{p}-L^{q}$ trace inequalities, J. London Math. Soc. 74 (2006), 497-511.

[5] C. T. DAT AND I. E. VERBITSKY, Finite energy solutions of quasilinear elliptic equations with sub-natural growth terms, Calc. Var. PDE 52 (2015), 529-546.

[6] DAT T. CAO AND I. E. VERBITSKY, Nonlinear elliptic equations and intrinsic potentials of Wolff type, J. Funct. Analysis 272 (2017), 112-165. 
[7] Dat T. CaO And I. E. Verbitsky, Pointwise estimates of Brezis-Kamin type for solutions of sublinear elliptic equations, Nonlin. Analysis, Ser. A: Theory, Methods \& Appl. 146 (2016), $1-19$.

[8] M. Frazier, F. NAZARov, AND I. Verbitsky, Global estimates for kernels of Neumann series and Green's functions, J. London Math. Soc. 90 (2014), 903-918.

[9] M. Frazier And I. Verbitsky, Positive solutions to Schrödinger's equation and the exponential integrability of the balayage, Ann. Inst. Fourier (Grenoble) (published online), arXiv:1509.09005

[10] O. Frostman, Potentiel de masses à somme algébrique nulle, Kungl. Fysiogr. Sällskapets i Lund Förhandlingar [Proc. Roy. Physiog. Soc. Lund] 20 (1950), 1-21.

[11] B. Fuglede, On the theory of potentials in locally compact spaces, Acta Math. 103 (1960), 139-215.

[12] E. Gagliardo, On integral trasformations with positive kernel, Proc. Amer. Math. Soc. 16 (1965), 429-434.

[13] A. GRIGOR'YAN AND I. E. VERBITSKy, Pointwise estimates of solutions to semilinear elliptic equations and inequalities, J. d'Analyse Math. (to appear) arXiv:1511.03188

[14] A. GRIGOR'YAN AND I. E. VERBITSKY, Pointwise estimates of solutions to nonlinear equations for nonlocal operators, arXiv:1707.09596

[15] W. Hansen And I. Netuka, On the Picard principle for $\Delta+\mu$, Math. Z. 270 (2012) 783807.

[16] M. MARCus And L. VÉron, Nonlinear Second Order Elliptic Equations Involving Measures, Walter de Gruyter, Berlin-Boston, 2014.

[17] B. MAUREY, Théorèmes de factorisation pour les opérateurs linéaires à valeurs dans les espaces $L^{p}$, Astérisque 11 (1974) Soc. Math. France, Paris.

[18] V. MAZ'YA, Sobolev Spaces, with Applications to Elliptic Partial Differential Equations, 2nd, Augmented Edition. Grundlehren der math. Wissenschaften 342, Springer, Berlin, 2011.

[19] S. QUINN AND I. E. VERBITSKY, Weighted norm inequalities of $(1, q)$-type for integral and fractional maximal operators, Harmonic Analysis, Partial Differential Equations and Applications, in Honor of Richard L. Wheeden, eds. S. Chanillo et al., Ser. Appl. Numer. Harmonic Analysis, Birkhäuser, 2017, 217-238.

[20] S. QUINN AND I. E. VERBITSKy, A sublinear version of Schur's lemma and elliptic PDE, Analysis \& PDE (to appear), arXiv:1702.02682

[21] E. SteIn AND G. WeISS, Introduction to Fourier Analysis on Euclidean Spaces, Princeton Univ. Press, Princeton, N.J., 1971.

[22] P. SzEPTYCKI, Notes on Integral Transformations, Dissert. Math. (Rozprawy Mat.), 231 (1984), p. 48.

Department of Mathematics, University of Missouri, Columbia, MO 65211, USA

E-mail address: verbitskyi@missouri.edu 